\section{Synchronous colorectal liver metastases: treatment dilemmas and hopes for improving oncological outcomes}

\author{
“There is no doubt that the management of patients with colorectal \\ cancer and synchronous colorectal liver metastases is complex. Many \\ factors must be carefully considered such as location and extent of \\ primary tumor, colorectal liver metastases and presence of symptoms in \\ order to adopt the 'ideal' therapeutic strategy."
}

\section{Georgios D Lianos¹ \& Georgios K Glantzounis ${ }^{*, 1}$}

First draft submitted: 3 February 2016; Accepted for publication: 9 March 2016; Published online: 29 March 2016

\begin{abstract}
Despite dramatic advances in cancer treatment, colorectal cancer (CRC) remains a major health problem. CRC constitutes the third most common malignancy in terms of incidence and fourth for cancer mortality worldwide [1]. Approximately $15-25 \%$ of patients with CRC present with synchronous colorectal liver metastases (CRCLM) [1-3]. In this editorial, we deal with synchronous CRCLM with an effort to summarize the potential and challenges of current therapeutic strategies. Treatment algorithms and expectations for improving oncological outcomes of these patients are also discussed.
\end{abstract}

\section{CRC \& CRCLM}

This type of cancer is the result of the accumulation of genetic modifications that lead to CRC carcinogenesis. Approximately $75 \%$ of CRCs occur in people without genetic background (sporadic cases) [3]. The liver is the most common site of metastases and in 70-80\% of cases, metastases are confined to the liver only. It is reported that 5-year survival rates of potentially complete surgical resection (R0) of
CRCLM are approximately 35-50\%. Unquestionably, the improvement of resection techniques and perioperative care has dramatically improved perioperative outcomes [4]. Currently, hepatic resection for CRCLM is safe and effective and when performed at high volume centers a perioperative mortality rate of $1 \%$ is achieved. However, high recurrence rates ( 60\%) suggest the crucial need for developing more effective systemic regimens in the near future $[4,5]$. An important issue in the CRC field concerns patients presenting with synchronous CRCLM [5].

\section{Synchronous CRCLM: definitions}

Despite the lack of randomized controlled trials (RCTs) to provide definitions, in accordance with the recent recommendations of the international multidisciplinary consensus on synchronous liver metastases from CRC, synchronous CRCLM are termed as liver metastases detected at or before diagnosis of the colorectal tumor [6]. Moreover, as early metachronous metastases are defined those detected within 12 months after diagnosis or surgery of the
'Department of Surgery, loannina University Hospital \& School of Medicine, University of loannina, loannina, Greece *Author for correspondence: Tel.: +30 265 109 9887; gglantzounis@uoi.gr

\section{KEYWORDS}

- colorectal cancer guidelines

- colorectal liver metastases

- liver-first strategy
“Approximately $15-25 \%$ of patients with colorectal cancer present with synchronous colorectal liver metastases." 
"Recently, there has been interest in another surgical option for patients with synchronous colorectal liver metastases.” colorectal tumor, late metachronous metastases are defined those detected more than 12 months after diagnosis or surgery of the primary tumor. It has to be highlighted that 'synchronous disease' is robustly associated with poor prognosis. Therefore, synchronous CRCLM are considered more 'aggressive' in terms of cancer biology. Notably, survival rates are lower in comparison with metachronous metastases and especially with late metachronous metastatic status [6].

\section{Treatment dilemmas of patients with} synchronous CRCLM

It is reported that the management of patients with synchronous CRCLM is complex and challenging [7]. The literature supports that multimodal treatment comprising surgery, chemotherapy and radiotherapy for rectal cancer, should be offered to these patients. The need for prompt surgical intervention for colorectal primary tumors and CRCLM leads to various surgical approaches. Treatment dilemmas are arising because of poor long-term outcomes of the conventional therapeutic strategy (primary-first approach). The other available treatment strategies for patient management are called 'reverse' strategies. To date, these treatment options are the primary-first approach, simultaneous resection and the liver-first approach [8].

The primary-first, or classical approach, includes resection of the primary tumor followed by chemotherapy and radiotherapy, especially for rectal tumors. Liver surgery is performed 3-6 months after the resection of the primary colorectal tumor if CRCLM are still operable [9]. This treatment strategy is plausible because primary colorectal tumors represent potential metastatic sources and are also a source of potential complications [9]. It is supported that the main advantage of this approach is that it avoids potential complications from the primary tumor and that could decrease the risk of progression of the primary tumor during hepatic resection or initial chemotherapy. The main disadvantage is the potential progression of CRCLM during the primary tumor surgical intervention. The progression of CRCLM to an unresectable status represents a dramatic prognostic event for each patient [10]. Notably, recent evidence indicates that only $\sim 30 \%$ of patients can successfully complete this approach due to the progression of metastases to an unresectable status [11].

Simultaneous resection of colorectal tumor and synchronous CRCLM presents a real surgical challenge. This option can be adopted with or without preoperative chemotherapy, while postoperative chemotherapy and radiotherapy for rectal malignancies is adopted after surgical treatment. This approach has been proposed in order to avoid the delay and potential progression of CRCLM. The main advantage of this strategy is that tumor resection takes place in a single surgical procedure [12]. On the other hand, this strategy is reported to be associated with significantly increased postoperative morbidity and mortality. Recent evidence suggests that simultaneous resections have postoperative morbidity rates of between 5 and $48 \%$ when patients undergo minor hepatectomies, while these rates are between 33 and 55\% when major hepatectomies are performed. For these reasons, the literature supports that simultaneous resection is suitable only for carefully selected patients $[12,13]$. It is recommended to perform rectal resection simultaneously with minor hepatectomy only or to perform major liver resection simultaneously mainly with right-sided colon resection [13].

Recently, there has been interest in another surgical option for patients with synchronous CRCLM. Taking into account that metastases represent the major cause of death of cancer patients, Mentha et al. [14] described the liverfirst strategy for the first time. In this surgical option, after a period of systemic chemotherapy, the CRCLM are resected before the colorectal tumor [14]. The liver-first approach is based on the concept that the main determinant for patient's survival is the presence of systemic disease. Preoperative systemic chemotherapy treats both primary symptoms (bleeding, mild obstruction) and metastases. It also provides a period of time that permits occult extrahepatic disease to be detected. In fact, this strategy could be called chemotherapy-first because the first approach is chemotherapy that does not affect negatively on resection of the colorectal carcinoma and synchronous liver metastases (LM), and may downstage LM believed to be unresectable [15]. Along with these effects on synchronous CRCLM, the chemotherapy could downstage the primary tumor. Patients with no obstructive CRC with extensive LM that necessitates downstaging may also benefit from this strategy, as it could provide a narrow window of resectability [15].

The first systematic review on this issue was reported by Jegatheeswaran et al. [16]. After a 
literature search, 417 studies were identified, of which only four described the liver-first approach and survival data. The authors concluded that a liver-first approach for patients with synchronous CRCLM is possible but is associated with a wide range of survival outcomes [16]. Therefore there is a need for well-designed studies comparing the liver-first approach with the conventional approach in order to reach safe conclusions. A recent meta-analysis on this issue by Kelly et al. [17] compares primary-first, simultaneous and liver-first approaches. A total of 18 studies with approximately 3600 patients were included in this study. Analysis of the 5-year overall survival showed no significant differences between these surgical options and perioperative mortality among these strategies was also not significant. However, recent evidence suggests that the liver-first approach is safe and can be a valid alternative option in patients with locally advanced colorectal carcinoma and synchronous CRCLM [6,15].

However, taking all of the above into consideration, we have to highlight that the recommended surgical treatment for patients with synchronous CRCLM still remains controversial [18].

\section{Recommendations based on current evidence}

In an effort to establish an algorithm for all clinical scenarios and to overcome treatment dilemmas, it seems that for asymptomatic CRC and resectable synchronous CRCLM, the current recommended option is for chemotherapy first, followed either by simultaneous surgery in cases with limited hepatic disease and easily resected primary tumors or by staged surgery for other patients [6,18]. Moreover, for asymptomatic CRC and unresectable synchronous CRCLM, the recommended option is chemotherapy-first, with the aim of making metastases resectable. Then liver resection can be performed first and resection of the primary tumor will follow. As for symptomatic CRC and resectable synchronous CRCLM, resection of the primary tumor must be performed followed by chemotherapy and then surgery of LM [6]. For symptomatic CRC and unresectable synchronous CRCLM, resection of the primary tumor for perforated or occlusive tumors should be performed, followed by chemotherapy and then surgery of metastases if it is possible [6,19]. Four courses of first-line optimal chemotherapy (with targeted therapy when it is possible) are recommended for synchronous unresectable liver metastases. Assessment of response every 2 months and a total duration of 6 months systemic therapy is considered necessary [6]. However, the role of monoclonal antibodies in resectable disease is not yet clear. It is hoped that the current expert consensus recommendations and the treatment plans for different clinical scenarios [6], as well as well-designed future RCTs, will help to improve oncological outcomes of patients with synchronous CRCLM [6,18-19].

The crucial role of multidisciplinary teams (MDT) in the effective management of patients with synchronous CRCLM has to be emphasized. It has been shown that specialized MDTs dedicated to the management of stage IV CRC, are superior to that of a general CRC-MDT [20]. These specialized MDTs should include surgeons specializing in colorectal, hepatobiliary and thoracic surgery, dedicated CRC medical and radiation oncologists, imaging and interventional radiologists and dedicated CRC pathologists $[6,20]$. Also the role of imaging in evaluating response to preoperative treatment is very important. Finally, histopathology also has a main role in the effective management of patients with synchronous CRCLM.

\section{Conclusion \& future perspective}

There is no doubt that the management of patients with CRC and synchronous CRCLM is complex [17-19]. Many factors must be carefully considered such as location and extent of primary tumor, CRCLM and presence of symptoms in order to adopt the 'ideal' therapeutic strategy. However, the best treatment strategy is still unclear because of limited amount of available data. A personalized treatment algorithm for each patient with synchronous CRCLM is necessary to be established after discussion among the MDT. Careful consideration of all surgical and medical options with the aim to avoid complications, achieving improved oncological outcomes, must be the gold standard of care for every patient [21]. A liver-first approach is safe and feasible in selected patients with synchronous CRCLM. It provides good longterm survival in patients with systemic extended disease but with high recurrence rate. Ongoing prospective controlled large-scale trials may contribute significantly to better definition of patient's selection criteria for the application of the most effective strategy. “...for asymptomatic colorectal cancer and resectable synchronous colorectal liver metastases, the current recommended option is for chemotherapy first, followed either by simultaneous surgery in cases with limited hepatic disease and easily resected primary tumors or by staged surgery for other patients." 
Financial \& competing interests disclosure The authors have no relevant affiliations or financial involvement with any organization or entity with a financial interest in or financial conflict with the subject matter or materials discussed in the manuscript. This includes employment, consultancies, honoraria, stock ownership or options, expert testimony, grants or patents received or pending, or royalties.

No writing assistance was utilized in the production of this manuscript.

\section{References}

1 Siegel RL, Miller KD, Jemal A. Cancer statistics, 2015. CA Cancer J. Clin. 65(1), 5-29 (2015).

2 Lianos GD, Glantzounis GK, Mangano A, Rausei S, Roukos DH. Colorectal liver metastases guidelines, tumor heterogeneity and clonal evolution: can this be translated to patient benefit? Future Oncol. 10(10), 1723-1726 (2014).

3 van Geel RM, Beijnen JH, Bernards R, Schellens JH. Treatment individualization in colorectal cancer. Curr. Colorectal Cancer Rep. 11(6), 335-344 (2015).

4 Dhir M, Sasson AR. Surgical management of liver metastases from colorectal cancer. J. Oncol. Pract. 12(1), 33-39 (2016).

5 Norén A, Eriksson HG, Olsson LI. Selection for surgery and survival of synchronous colorectal liver metastases; a nationwide study. Eur. J. Cancer 53, 105-114 (2015).

6 Adam R, de Gramont A, Figueras J et al. Managing synchronous liver metastases from colorectal cancer: a multidisciplinary international consensus. Cancer Treat. Rev. 41(9), 729-741 (2015).

7 Adam R, De Gramont A, Figueras J et al. The oncosurgery approach to managing liver metastases from colorectal cancer: amultidisciplinary international consensus. Oncologist 17(10), 1225-1239 (2012).

8 Baltatzis M, Chan AK, Jegatheeswaran S, Mason JM, Siriwardena AK. Colorectal cancer with synchronous hepatic metastases: systematic review of reports comparing synchronous surgery with sequential bowel-first or liver-first approaches. Eur. J. Surg. Oncol. 42(2), 159-165 (2015).

9 Castellanos JA, Merchant NB. Strategies for management of synchronous colorectal metastases. Curr. Surg. Rep. 2, 62 (2014).

10 Brouquet A, Nordlinger B. Surgical strategies to synchronous colorectal liver metastases. Dig. Dis. 30 (Suppl. 2), 132-136 (2012).

11 Brouquet A, Mortenson MM, Vauthey JN et al. Surgical strategies for synchronous colorectal liver metastases in 156 consecutive patients: classic, combined or reverse strategy? J. Am. Coll. Surg. 210(6), 934-941 (2010).

12 Mayo SC, Pulitano C, Marques $\mathrm{H}$ et al. Surgical management of patients with synchronous colorectal liver metastasis: a multicenter international analysis. J. Am. Coll. Surg. 216(4), 707-716 (2013).

13 Ihnát P, Vávra P, ZonČa P. Treatment strategies for colorectal carcinoma with synchronous liver metastases: which way to go? World J. Gastroenterol. 21(22), 7014-7021 (2015).

14 Mentha G, Roth AD, Terraz S et al. 'liver-first' approach in the treatment of colorectal cancer with synchronous liver metastases. Dig. Surg. 25(6), 430-435 (2008).

15 Waisberg J, Ivankovics IG. Liver-first approach of colorectal cancer with synchronous hepatic metastases: a reverse strategy. World J. Hepatol. 7(11), 1444-1449 (2015).

16 Jegatheeswaran S, Mason JM, Hancock HC, Siriwardena AK. The liver-first approach to the management of colorectal cancer with synchronous hepatic metastases: a systematic review. JAMA Surg. 148(4), 385-391 (2013).

17 Kelly ME, Spolverato G, Lê GN et al. Synchronous colorectal liver metastasis: a network meta-analysis review comparing classical, combined, and liver-first surgical strategies. J. Surg. Oncol. 111(3), 341-351 (2015).

18 Siriwardena AK, Mason JM, Mullamitha S, Hancock HC, Jegatheeswaran S. Management of colorectal cancer presenting with synchronous liver metastases. Nat. Rev. Clin. Oncol. 11(8), 446-459 (2014).

19 de Mestier L, Manceau G, Neuzillet C et al. Primary tumor resection in colorectal cancer with unresectable synchronous metastases: a review. World J. Gastrointest. Oncol. 6(6), 156-169 (2014).

20 Jones RP, Vauthey JN, Adam R et al. Effect of specialist decision-making on treatment strategies for colorectal liver metastases. Br. J. Surg. 99(9), 1263-1269 (2012).

21 Lykoudis PM, O'Reilly D, Nastos K, Fusai G. Systematic review of surgical management of synchronous colorectal liver metastases. $\mathrm{Br}$. J. Surg. 101(6), 605-612 (2014). 